\title{
MAGNETIC-MECHANICAL ACCUMULATOR OF KINETIC ENERGY
}

\author{
Jan Valtera, Jaroslav Beran \\ Technical University Liberec, Faculty of Mechanical Engineering, Department of Textile and Single-Purpose Machines, \\ Studentska 2, 46117 Liberec, Czech Republic \\ e-mail: jan.valtera@tul.cz, jaroslav.beran@tul.cz
}

\section{Abstract:}

The paper focuses on the process of non-contacting kinetic energy accumulation at the dead-end positions of a reciprocating rectilinear motion with a variable stroke. It describes the development of a magnetic-mechanical accumulator that absorbs energy while the moving part is decelerating towards the dead-end position and releases the energy back to the system while it is accelerating from the dead-end position. At the same time, it enables stroke modification of a certain level with a minimal impact on the maximum force of the accumulator. With respect to the magneto-static analysis of components, the preload of the mechanical element has been set accordingly. The dynamic analysis of the system has been carried out and the prototype produced and tested on the testing rig.

\section{Keywords:}

Energy, accumulation, reciprocation, magnetic force, stroke

\section{Introduction}

The reciprocating rectilinear motion with variable stroke is used in many mechanical systems. In the textile field, it is used on rotor spinning machines in order to guide yarn while winding it up to the cross-wound packages. The trend in increasing operating speeds brings heavy demands on the strength of elements as they are exposed to substantial amounts of dynamic forces [1]. An increase of deformation and vibration of elements can contribute to faults in the system [4]. Therefore, the usage of either light-weighted elements or systems of an energy accumulation is taken into account. The aim of the kinetic energy accumulator is to reduce dynamic effects upon the system. This can be achieved by the energy absorption while the translational element is decelerating towards the dead end of its motion and by the release of this energy back to the system while it is accelerating from the dead end. In order to reduce dynamic effects, the minimal mass of any required additional moving element should be taken into consideration. The kinematic scheme in the Figure 1 describes a specific reciprocating rectilinear motion that is derived from the traversing system of the rotor spinning machine [2]. In this system, the output motion $\mathrm{Z}(\varphi)$ is comprised of the principal motion $S 1(\varphi)$ and the additional one $S 2(\varphi)$. Figure 2 represents characteristic courses of the principal motion $S 1(\varphi)$ and its derivatives, and the additional motion $S 2(\varphi)$, with respect to the virtual cam angle $\varphi$.

Due to an increase of dynamic effects on the dead ends of the translational motion, several solutions of energy accumulation have been devised. The system described in [7] consists of a pair of preloaded springs pushing against the moving part at its dead end. However, from the standpoint of vibration, it is convenient to accumulate the dynamic forces in a non-contacting way to avoid any additional vibration excitation. The magnetic accumulation with additional motion [10] introduces a sophisticated solution, where the permanent magnets and additional driven support is used to accumulate the kinetic energy at the dead ends of the motion. However, the newly developed magnetic-mechanical accumulator [9] introduces a simple way of non-contacting reduction of dynamic forces upon the translational part of the system without using any additional driver.

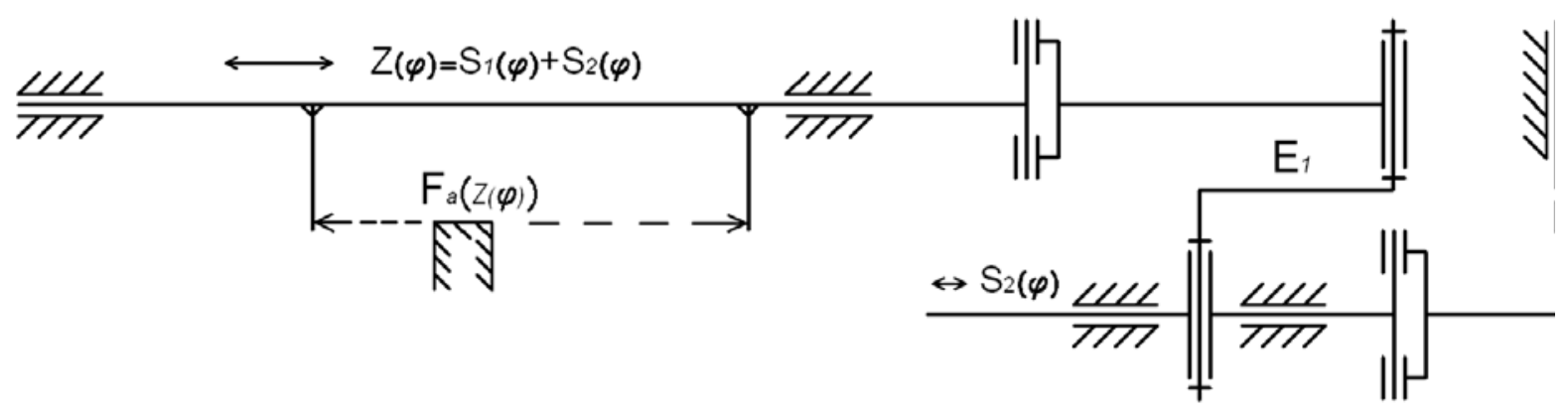

Figure 1. The kinematic scheme of the reciprocating motion with the variable stroke. 

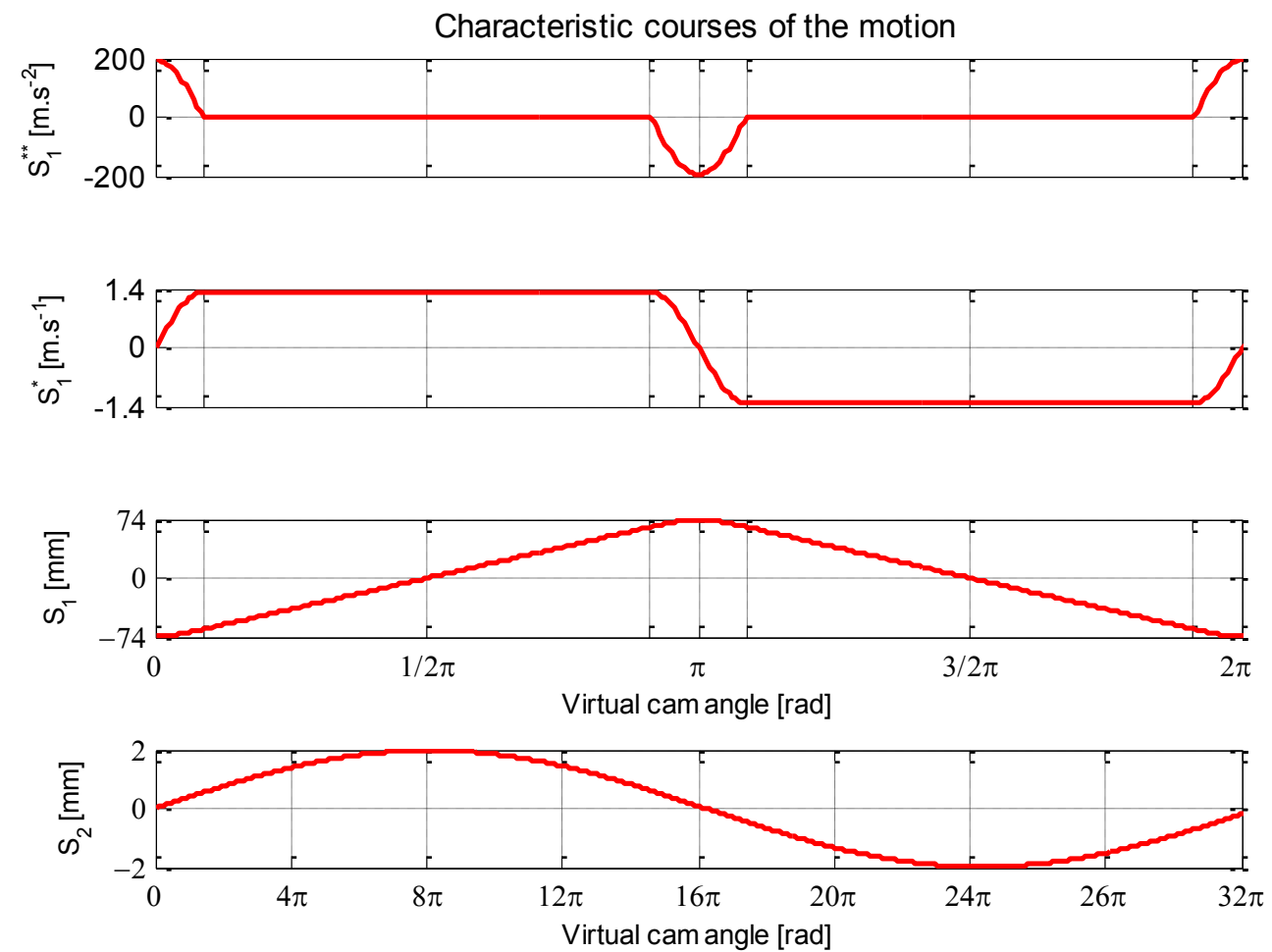

Figure 2. Characteristic courses of the reciprocating motion.

\section{Specification of devised magnetic-mechanical accumulator}

The principle of the developed magnetic-mechanical accumulator is based on the law of energy conservation [8], where the kinetic energy Ekin caused by dynamic forces is converted into the potential energy of repelling magnets Emag and potential energy of an elastic object Emech. For isolated systems [6], the principle can be described by formula 1 .

$$
E_{\text {kin }}=E_{\text {mag }}+E_{\text {mech }} \text {. }
$$

The force courses of the proposed concept with magnetic and mechanical elements are shown in the Figure 3. It describes the progressive increase of repelling magnetic force with comparison with a linear increase of the spring force. At the moment, when the magnetic force is equal to the preload of the spring, the further progressive magnetic force is transferred to compression of the spring. The overall force exerted by the accumulator is called Fa. In order to maintain the minimal difference of Fmax - Fmin, the spring is characterised by a low stiffness. By means of this, the stroke modification within certain distance affects the maximal accumulated force insignificantly. The repelling magnetic force that is shown in Figure 3 was obtained from a magneto-static analysis of neodymium magnets with the proposed geometry. With respect to this, the parameters of the spring can be set accordingly.

According to the law of energy conservation [8], the simplified formula 1 can be further expressed as a work of the system by means of forces acting along the translational displacement $Z$ as:

$\int_{0}^{Z} F_{d y n} \cdot d Z=\int_{0}^{S_{\max }-S_{2 \max }} F_{m a g} \cdot d Z+\int_{S_{\max }-S_{2 \max }}^{Z} F_{\text {mech }} \cdot d Z$, where $Z \in\left\langle 0, S_{1_{\max }}+S_{2}\right\rangle$.
According to [3], the transition section of the translational motion (reversion section) corresponds to virtual cam angle $16^{\circ}$, with a respective displacement of $9 \mathrm{~mm}$, whereas the additional motion maximum $S 2$ max is considered to be within 2 $\mathrm{mm}$. As a result, magnets absorb most of the kinetic energy at higher speeds and the spring is activated at lower speed only. This enables minimising the translational momentum effect of the accelerated mechanical components sufficiently.

Supposing the maximum of the additional motion $S 2 m a x=2 \mathrm{~mm}$, parameters of the proposed accumulator design were carried out. In order to accommodate the elastic linear spring with a low rate, the proposed concept is designed in a symmetrical arrangement (see Figure 4). This concept allows accumulation at both dead-end positions, while using single elastic spring. The translational rod 0 is slide-free connected to the ground, whereas the accumulator frame 9 is fixed to the ground. The cylindrical-shaped magnets $1 L$, resp. $1 R$ are fixed to the rod. While the rod is translating towards the right dead end, magnets $1 L$ and $2 L$ approach each other. As they are axially magnetised with the same poles towards each other, their repelling force increases. The magnet $2 L$ is fixed to the slider $3 L$, which is slidefree connected to the accumulator frame. Initially, the slider is pushed against the edge of the frame by the preloaded force of the spring 4 . The force preload is set in order to maintain a sufficient clearance between magnets $1 L$ and $2 L$ at the force equilibrium. This prevents any damage caused by an eventual contact between magnets. At the transition point, the moment when the magnetic repulsive force is equal to spring preload $\left(Z=S_{1 \max }-S_{2 \max }\right)$, further translational motion is transmitted to the translation of the magnet $2 L$ with the slider $3 L$. Thanks to this, the progressive increase of repelling force is transferred to a linear increase of the spring force. 


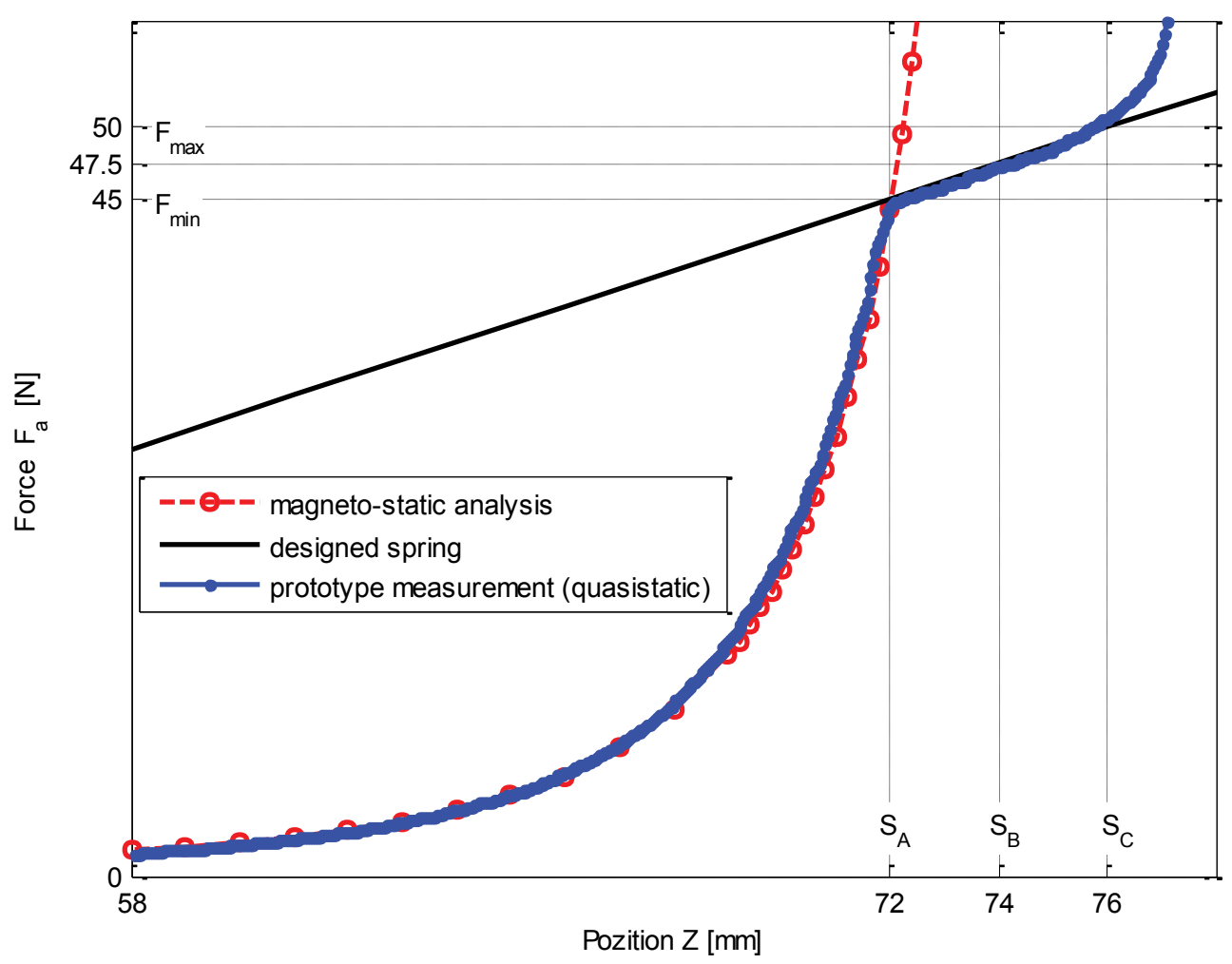

Figure 3. Specification of accumulated force along the position of the translationalelement.

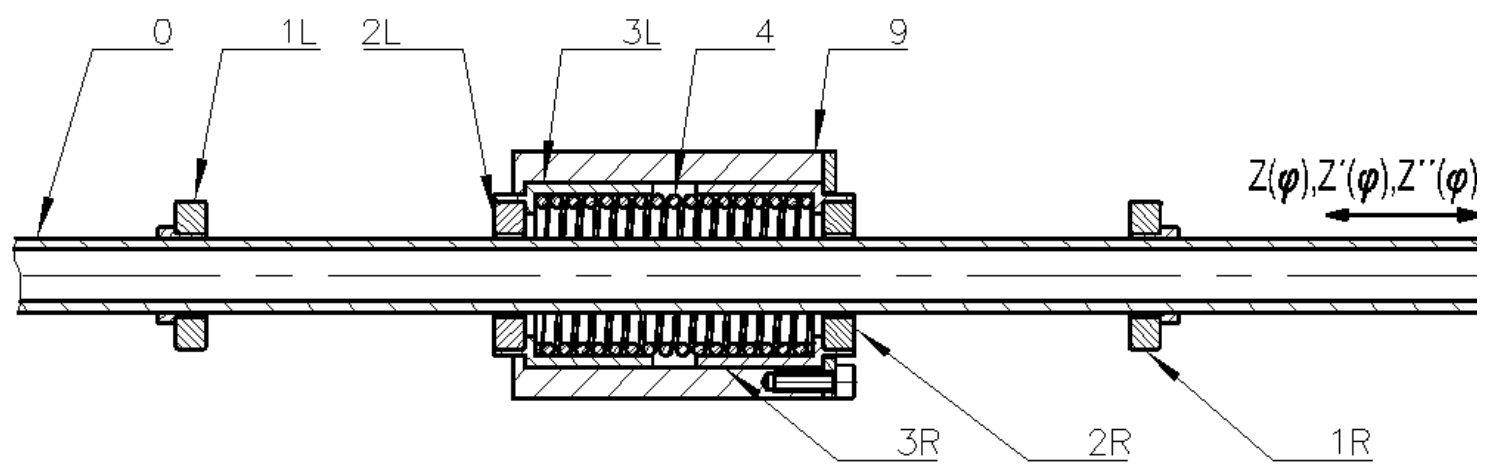

Figure 4. Schematic view of the proposed design.

\section{Analyses and testing}

During the prototype design process, several analyses and tests were carried out. Parameters $S 1$ and $S 2$ were specified according to Figure 2. The geometry and material grade of permanent magnets has been optimised with regard to repelling magnetic force and overall weight of magnets. There are several systems for calculating the magnetic force available [5]. The magneto-static analysis was carried out in the software FEMM [6] as a $2 \mathrm{D}$ axisymmetric task with variable distance of magnets. Based on this, the calculated spring stiffness $k=1.25 \mathrm{~N} / \mathrm{mm}$ and the spring preload Fmin=45 N were determined. These settings of both magnets and spring parameters provide sufficient clearance between magnets at the point of force equilibrium. In order to simulate the dynamic behaviour of the system, a model with a translational element and magnetic-mechanical accumulator was devised in the software Adams/View. It utilises the high-stiff translational rod made out of carbon composite [11] and force defined by external tabular form related to actual position of particular rod element. The overall weight of magnets and clamping elements which are fixed to the translation rod is $23.2 \mathrm{~g}$. The dynamic analysis of the system was carried out in the software ADAMS/View for motion frequencies $0.5 \div 4 \mathrm{~Hz}$. The accumulate force of the manufactured prototype was measured on the testing rig and is shown in Figure 3. Figure 5 shows the results from translational testing rig that was equipped with one accumulator. The force has been measured at the point where the translational element is connected to the mechanism, generating the required motion course. The detailed view of the transition section of the motion is shown in Figure 6.

Overall results from the system analyses as well as from the testing proved both the appropriate parameters setting and functionality of the prototype. However, the dynamic behaviour 


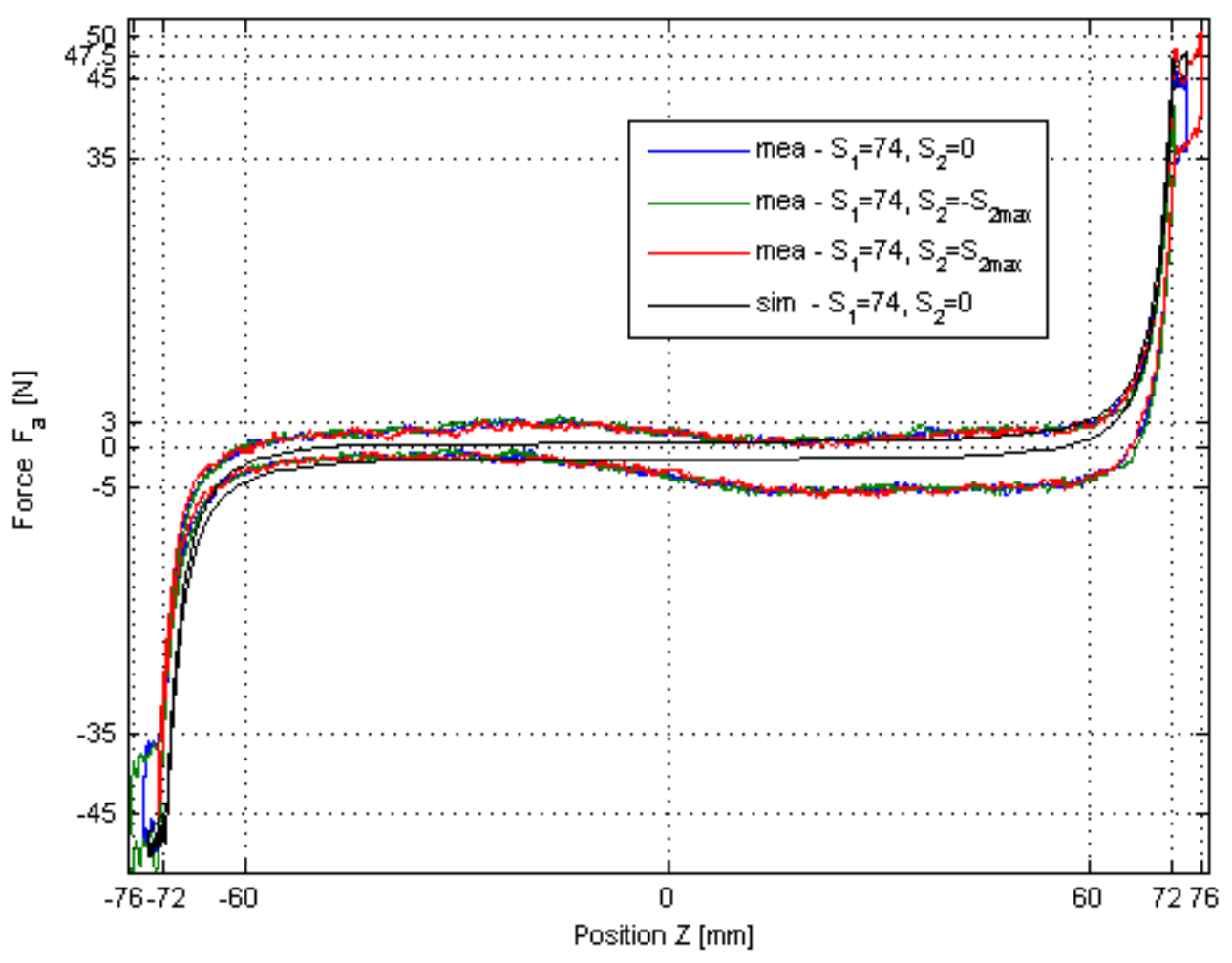

Figure 5. Prototype measurement.

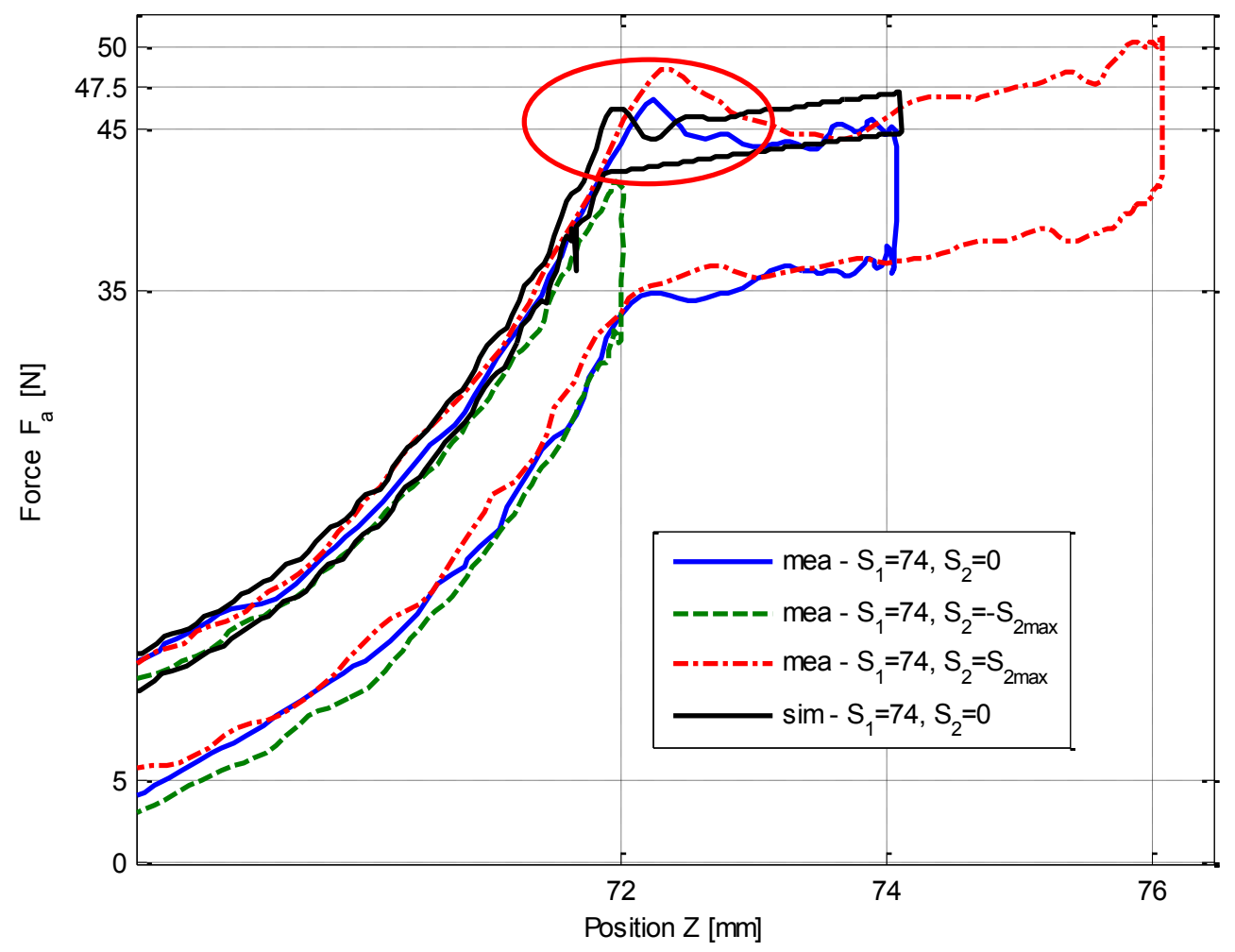

Figure 6. Prototype measurement - detailed view.

of sliding parts of the accumulator affects the exerted force considerably. For more precise system simulation, the dynamic force of the accumulator sliding parts should be taken into account. Furthermore, the magneto-static analysis of repelling magnets with a radial displacement should be carried out in order to describe potential misalignment of translational parts and parts of the accumulator and its effect on the magnetic force deviation.

\section{Evaluation}

The maximal value of the measured force, shown in Figures 5 and 6 , corresponds to both theory and simulation. However, results indicate considerable amount of the friction at the testing rig in comparison with simulation. Moreover, at dead-end area, this value is extended up to $10 \mathrm{~N}$. This suggests a 
misalignment of magnets and translational element, which may give rise to transverse force components. The measurement however proved the functionality and proper parameters settings of the devised accumulator.

The force increase at the transition point, as highlighted in Figure 6 , is related to translational momentum of the accelerating accumulator magnet, slider and several spring coils. This affects the force course and dynamic behaviour inappropriately.

The overall increase of exerted force due to the $S 2$ variation is evaluated and expressed by means of an effective force Feff, which is determined according to the following equation:

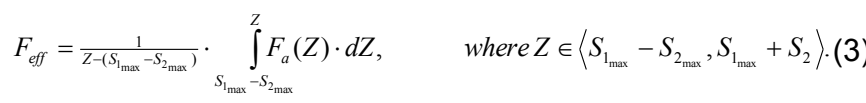
(3)

Based on the results from the dynamic analysis and equation (3), the variation of Feff with respect to different values of the parameter $S 2$ has been determined from the simulation and the results are shown in Figure 7 . The course $S 2=2$ describes the maximal compression of the spring. On the contrary, the course $S 2=-2$ represents the state when the dead end is reached just at the transition point and spring element is not pressed at all. At lower frequencies, the slight decrease is present in this course. This is related to deformation of the non-rigid translational rod used in the simulation, which is compressed by exerted magnetic force, and therefore it does not reach the theoretical dead-end position.

From Figure 7, it is evident that the effective force Feff related to the $S 2$ variation changes together with the motion frequency. The magneto-static analysis force course in Figure 3 suggests the potential increase in the effective force due to $S 2$ variation of a system without additional elastic element used. Therefore, the force deviation (maximum of $7 \mathrm{~N}$ ) confirmed the assumption of force reduction of the devised system upon the variation of the translational motion. The system is especially useful for the non-contacting accumulation of kinetic energy in mechanical systems with a stroke modification, where the force (stress respectively) at the dead-end position is required to keep a certain level. The devised system proved the functionality and can be easily modified in order to meet the requirements of a particular application.

\section{Conclusions}

Based on the magneto-static and dynamic analysis, parameters of the new devised accumulator were determined and set. The prototype was manufactured in the symmetrical arrangement with minimal additional weight to be fixed to the translational rod. The system proved the ability to transmit the progressive increase of magnetic force into the linear low rate spring force and therefore eliminate the stroke variation sufficiently. The measurement verified the satisfying functionality of noncontacting energy accumulation with the stroke variation $S 2<-2.2>$. From the results, it is evident that at low motion frequencies the effective force varies within $2.5 \mathrm{~N}$ and satisfies the proposed requirements. The system stability is present up to a frequency of $2.5 \mathrm{~Hz}$ at which the overall effective force change remains within the acceptable value of $6.5 \%$ of the nominal value. At a frequency of $4 \mathrm{~Hz}$, results indicate that dynamic forces affect the system behaviour considerably, and the force increases up to $52.5 \mathrm{~N}$ (16\% of the nominal value). For higher motion frequencies, a further improvement might be required. The system proved its ability to accumulate kinetic energy within a certain stroke modification. The level of accumulated force can be modified optionally by the spring stiffness. Due to its modest design, it is also an effective solution in a multiple serial usage along translational element, which incorporates the force enlargement as well as the eventual longitudinal vibration reduction.

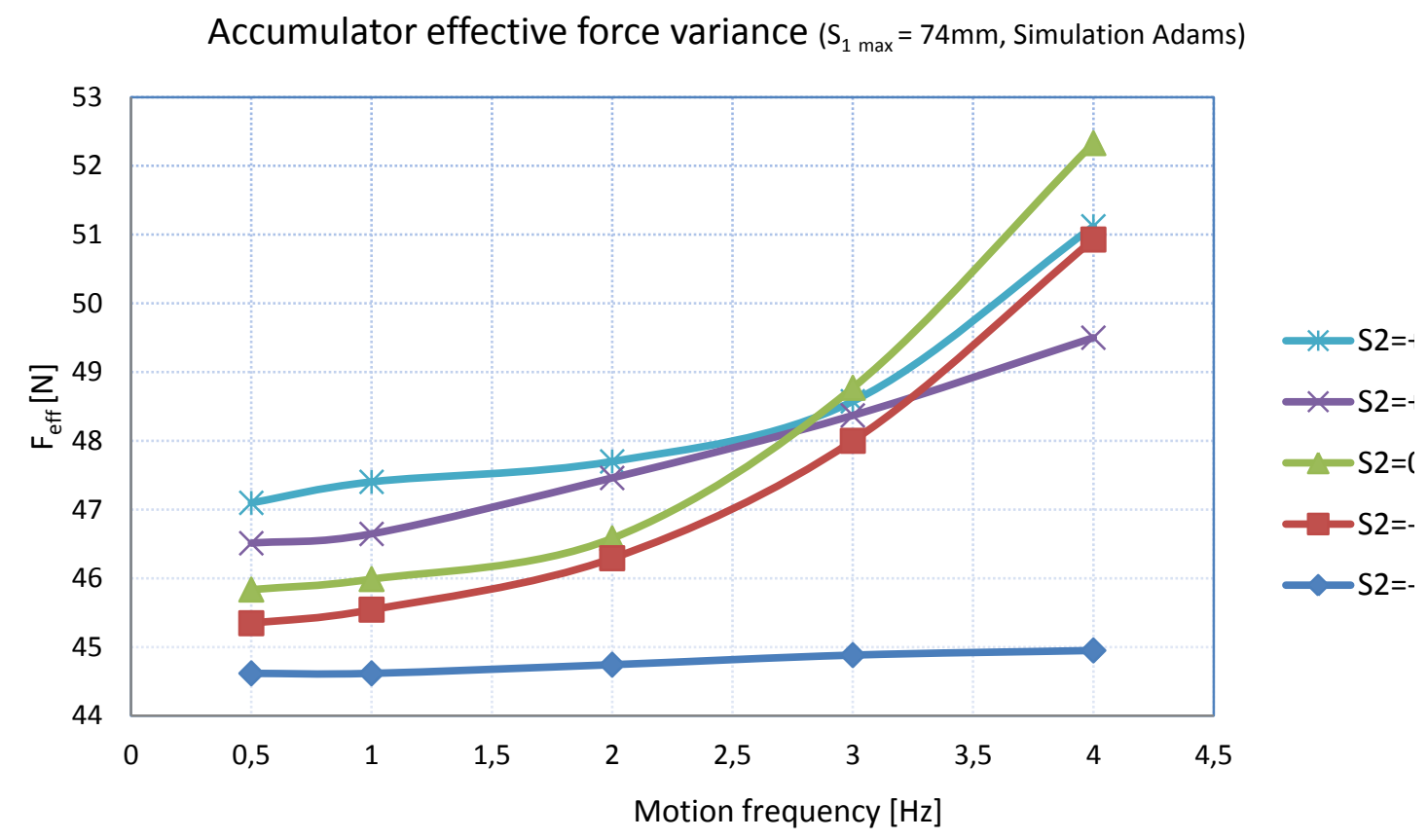

Figure 7. Effective force of the accumulator. 


\section{Acknowledgment}

The research reported in this paper was supported in part by the Project OP VaVpl Centre for Nanomaterials, Advanced Technologies and Innovation CZ.1.05/2.1.00/01.0005.

The paper has been elaborated with the financial support of Technical University of Liberec in the framework of the specific university research competition.

\section{References}

[1] BERAN, J., VALTERA, J.: Dynamic analysis of the distribution rod on the rotor spinning machine, In Proceedings of The XIIth Romanian Textile and Leather Conference Cortep 2007, Iasi, Romania, 2007, pp 367, ISSN: 15826392.

[2] BERAN, J., VALTERA, J., ZABKA, P., New trends in yarn distribution on spinning machines, In Proceedings of Srutex 2008, Liberec, Czech Republic, pp 579 ISBN 978/80-7372-418-4.

[3] HUBALEK, M., Novy system rozvadeni prize na rotorovych dopradacich strojich s vyuzitim servopohonu. Liberec, 2006. pp 98. Doctoral thesis at The Technical University of Liberec, Czech Republic.( in Czech).
[4] Idzik, M. (2009). Computer analysis distribution of the yarn linear density from open end spinning machine. AUTEX Research Journal, Vol.9, No4.

[5] Kosek, M., Mikolanda, T., et al. (2007). Effective and robust calculation of magnetic force. Procedings of Conference Technical Computing Praque 2007.

[6] Meeker, D., C., Finite Element Method Magnetics, Version 4.2. (11Apr2012), http>//www.fem.info.

[7] OERLIKON TEXTILE GMBH \& CO KG and BUTSCHKO SVEN. Cross/winding device for a textile machine which produces cross/wound bobbins. Inventors: BUTSCHKO Sven. International patent WO2008058605 A1. 22.5.2008.

[8] Serway, Jewett: (2010). Physics for scientists and engineers with modern physics. (8th ed.). Belmont, USA: Brooks/Cole.

[9] TECHNICAL UNIVERSITY OF LIBEREC and RIETER CZ s.r.o.. Method and device for traversing of yarn on textile machines. Inventors: BERAN Jaroslav and VALTERA Jan. European patent EP2567918 A2. 29.3.2012.

[10] VUTS a.s. and RIETER CZ s.r.o, Device for traversing of yarn wound-up on the bobbin. Inventors: FOUNE Frantisek and PUSTKA Martin. European patent EP2562112 A1. 27.2.2013.

[11] VALTERA, J., BERAN, J., Mathematical model of the long traversing rod with a discrete flexible link utilization, In: Proceedings of Transfer 2011 [CD-ROM], Trencin, Slovakia, November 23-24, 2011, ISBN:978-80-8075-454-9. 